\title{
Phenology of a tropical dry deciduous forest of Bhadra wildlife sanctuary, southern India
}

\author{
Appaji Nanda ${ }^{1,2^{*}}$, Hebbalalu S Suresh ${ }^{3}$ and Yelugere L Krishnamurthy ${ }^{1}$
}

\begin{abstract}
Introduction: This paper describes the leafing, flowering and fruiting phenology of canopy trees in the dry deciduous forest of Bhadra wildlife sanctuary from June 2004 to May 2006.

Method: All the woody canopy individuals ( $>20 \mathrm{~cm}$ girth at breast height) were identified and tagged with a unique number along a transect of approximately $2 \mathrm{Km}$ comprising 157 individuals of 22 species. Observations were made at monthly intervals from June 2004 to May 2006 for leafing, flowering and fruiting phenophases.

Result: Leaf fall starts in September, with a peak in December and January. Leaf initiation begins in February, with a peak in April before the monsoon. Leaf expansion starts in February from pre-monsoon with a peak in May and July during the monsoon. Leaf senescence begins in September to November and peaks in January to March. Flower bud initiates in January with a peak in April and May, and pollination begins in April with a peak in May and July before the monsoon to onset of monsoon. Fruit bud initiates in May with a peak in September and October. Unripened fruit was observed in May with a peak in September and November. Fruit fall begins in November with a peak in March.

Conclusion: Our results show that leafing and flowering activities occur in the summer or pre-monsoon. Fruiting patterns occur during the monsoon to post - monsoon season. Seasonality among various phenophases indicates that leaf senescence flower initiation and fruit fall have strong seasonality.
\end{abstract}

Keywords: Bhadra wildlife sanctuary; Dry deciduous forest; Phenophases; Rayleigh's Z test; Seasonality

\section{Introduction}

Global climate change is a reality and a continuous process that needs to be taken seriously, even though there are large uncertainties in its spatial and temporal phenological response of all ecosystems. Tropical deciduous forests in India account for approximately $46 \%$ of the forest land in the country (Singh and Singh 1998). Tropical forests exhibit a wide variation in patterns of vegetative and reproductive phenology on both large and small geographic scales (Morellato et al. 2000). Phenology is the study of the periodicity or timing of recurring biological events. What causes the timing of tropical deciduous forests with regard to biotic and abiotic forces, and does the

\footnotetext{
* Correspondence: nandabotany@gmail.com

${ }^{1}$ Department of Postgraduate Studies and Research in Applied Botany, Bioscience Complex, Jnana Sahyadri, Kuvempu University, Shankaraghatta, Shimoga 577 451, Karnataka, India

${ }^{2}$ Biodiversity Education and Research Laboratory, Environmental Study

Centre, Santhekaduru, Shimoga 577 222, Karnataka, India

Full list of author information is available at the end of the article
}

timing affect interrelation among phases of the same or different species (Sakai 2001)?

In tropical dry forests, phenological changes are caused by seasonal variations in rainfall (Daubenmire 1972; Borchert 1994a; Bullock and Solis-Magallanes 1990; Eamus and Prior 2001); stem water status, such as soil water availability; endogenous factors, such as leaf age and area, root size and distribution, and stem wood density (Borchert 1994b, 1998); photoperiod (Bullock and Solis-Magallanes 1990; Borchert et al. 2004; Rivera et al. 2002; Elliot et al. 2006); changes in temperature (Ashton et al. 1988; Williams-Linera 1997); and irradiance (Wright and van Schaik 1994). In contrast, biotic factors, such as competition for pollinators or pollinator attraction (Robertson 1895; Janzen 1967; Gentry 1974; Stiles 1975; Augspurger 1981; Appanah 1985; Murray et al. 1987; Sakai et al. 1999), competition for seed dispersers (Snow 1965), and avoidance of herbivory (Marquis 1988; Aide 1993; van Schaik et al. 1993; Coley and Barone 1996) have been interpreted as ultimate causes responsible for phenological patterns in

\section{实}


tropical species. Spatial and temporal variations in foliar phenology play a significant role in growth and reproduction of plant species (Suresh and Sukumar 2011).

Quantitative phenological studies and research that elucidates the levels of asynchrony among individuals and between species will help to understand the impact of climate change on tropical trees, as well as the potential consequences of these changes on the ecological community as a whole (Singh and Kushwaha 2005). The seasonality of tropical tree phenology is mainly determined by the duration and intensity of seasonal drought (Mooney et al. 1995); even conspecific trees often experience differing degrees of drought stress (Singh and Kushwaha 2005) and resource availability for consumer animals (Morellato et al. 2000). However few reports are available on seasonality studies in India (Singh and Singh 1992; Murali and Sukumar 1993, 1994; Bhat 1992; Prasad and Hegde 1986). With exception of the dry forest, montane forests in Niligiri have been reported in seasonality studies (Suresh and Sukumar 2011). In this paper we analyzed the phenological pattern in relation to rainfall, temperature, and circular statistics using the various phenophases and dates of observation to address:1) phenology of various tree species; 2) analysis of factors controlling phenology; and 3) seasonality of various phenophases.

\section{Methods}

\section{Study area}

Bhadra wildlife sanctuary is located in the Chikmagalur and Shimoga districts $\left(13^{\circ} 25^{\prime}\right.$ and $13^{\circ} 50^{\prime} \mathrm{N}, 75^{\circ} 15^{\prime}$ and $\left.75^{\circ} 50^{\prime} \mathrm{E}\right)$ of Karnataka, Central Western Ghats, India. The study was conducted in the Umblebailu region $\left(13^{\circ} 46^{\prime}\right.$ to $13^{\circ} 52^{\prime} \mathrm{N}, 75^{\circ} 36^{\prime}$ to $75^{\circ} 42^{\prime} \mathrm{E}$ ) of the dry deciduous forest. The terrain is gently undulating with valleys and steep hillocks, and a detailed geological account of the sanctuary has been previously described (Parameshwar 1996). The altitude is 690 to $750 \mathrm{~m}$ above mean sea level. Rainfall and temperature data for the study area were collected from the meteorological station, which is $5 \mathrm{~km}$ from the study site. Average monthly annual rainfall and temperature patterns during the study period are given in Figure 1.

In Champion and Seth (1968) dry deciduous forests are classified as southern dry mixed deciduous forests. The characteristic tree species are Terminalia paniculata, Anogeissus latifolia, Acrocarpus fraxinifolius, Haldina cordifolia, Bombax malabaricum, Dalbergia latifolia, Lagerstroemia lanceolata, Mitragyna parviflora, Pterocarpus marsupium, Terminalia bellerica, Ficus benghalensis, Lannea coromandelica, and Melia dubia. Quantitative descriptions of the vegetation are provided in Krishnamurthy et al. (2009, 2010) and Prakasha et al. (2008).

The climate is monsoonic with marked seasonal variations in temperature and rainfall. Depending on the variation in temperature, three seasons are observed in the area, namely pre-summer (November to January), summer (February to May), and rainy season (June to October). The cold or winter season starts from November and lasts until February with comparatively lower temperatures (15 to $19^{\circ} \mathrm{C}$ ) and significantly less rainfall. The rainy season starts in the second part of May with interrupted showers, and incessant rain begins in June, continues until September, and ends in the first part of November. During the summer the temperature ranges from 30 to $35^{\circ} \mathrm{C}$. July and August are normally the most precipitated months, receiving about $50 \%$ of the annual rainfall.

\section{Phenological observation}

All the woody canopy individuals $(>20 \mathrm{~cm}$ girth at breast height) were identified and tagged with a unique number along a transect of approximately $2 \mathrm{~km}$, comprising 157 individuals of 22 species. The identified individual species were confirmed using various regional floras (Yoganarasimhan et al. 1982; Saldanha 1996; Gamble and Fischer 1998; Ramaswamy et al. 2001; Neginhal 2004). Observations were made at monthly intervals from June 2004 to May 2006 for leafing, flowering, and fruiting phenophases. Binocular observations were made to check overlapping of events and tree branches. The leafing phenophases include different categories such as: 1) leafless stage; 2) leaf initiation; 3) leaf expansion; and 4) leaf senescence. Flowering phenophases categories include: 1) flower bud; 2) open flower; and 3) pollinating flower. Fruiting phenophases categories include:1) fruit bud; 2) immature fruit/ unripened fruit; 3)matured fruit/ripened fruit; and 4) fruit senescence. Each stage in the different categories of phenology was scored qualitatively with respect to both spread and intensity on a 0 to $100 \%$ scale.

\section{Data analysis}

Spearman's rank correlation with current and lag months was performed to assess the independent influence of each environmental factor using procedures described by Zar (2007).

\section{Seasonality study}

Seasonality was determined with circular statistics. Circular statistical analyses were conducted using the phenological variables and dates of observation. To calculate the circular statistical parameters, months were converted to angles, from $0^{\circ}=$ January (number 1 ) to $330^{\circ}=$ December (number 12 ) at intervals of $30^{\circ}$.

We converted the day of observation in a given month to angles. We used these angles and the number of species in a given month in a given phenophase to estimate Rayleigh's Z test interpretation:

$$
\mathrm{a}=360^{\circ} \mathrm{x} / \mathrm{k}
$$




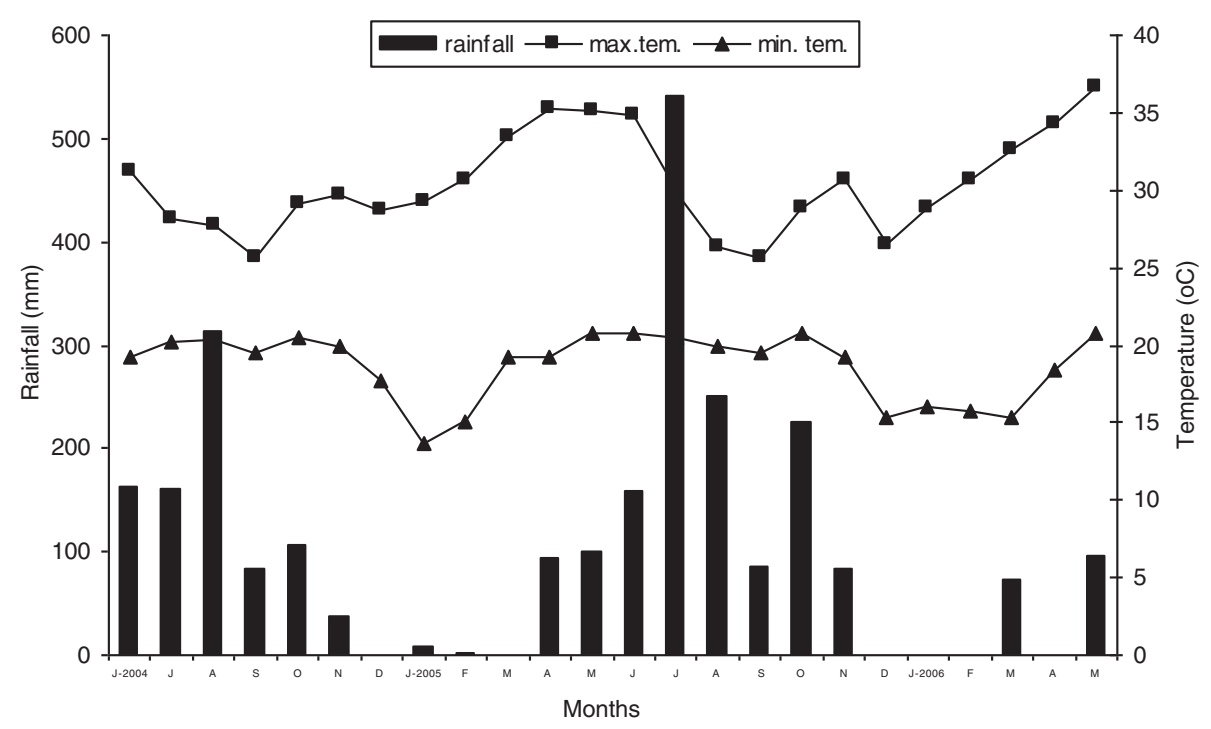

Figure 1 Total monthly rainfall $(\mathrm{mm})$ and mean monthly maximum and minimum temperature $\left({ }^{\circ} \mathrm{C}\right)$ at the dry deciduous forest of Bhadra wildlife sanctuary during the study period.

Where $\mathrm{a}$ is the angular direction (in degrees), $\mathrm{x}$ is the conversion of the date of observation from months to days, and $\mathrm{k}$ is the number of days in the year $(\mathrm{k}=365$, or 366 in a leap year).

The frequency of occurrence of species at each phenological variable within each angle was calculated and the following parameters were estimated for each study site: the mean angle a, the angular dispersal, confidence limits of the frequency distribution for each phenological variable, and vector $r$, a measure of concentration around the mean angle.

\section{Testing for the occurrence of seasonality}

The mean angle a, or mean date, is the time of year around which the dates of a given phenophase occurred for most species. Rayleigh' sZ test determines the significance of the mean angle. The hypotheses tested were: $\mathrm{H}_{\mathrm{O}}=$ dates are distributed uniformly (or randomly) around the circle or year; there is circular uniformity or no mean direction, and consequently, no seasonality; and $\mathrm{H}_{\mathrm{A}}=$ dates are not distributed uniformly around the year; there is a significant mean angle or mean direction, and consequently, there is some seasonality. If $\mathrm{H}_{\mathrm{A}}$ is accepted, the intensity of concentration around the mean angle, denoted by r, can be considered a measure of the degree of the seasonality. The vector $r$ has no units and may vary from 0 (when phenological activity is distributed uniformly throughout the year) to 1 (when phenological activity is concentrated around one single date or time of year). If $\mathrm{H}_{\mathrm{O}}$ is not rejected, then $\mathrm{r}=0$ (Morellato et al. 2000; Zar 2007).

\section{Results}

\section{General phenology}

Among the 22 species, leaf flush or leaf initiation becomes more pronounced from February (seven species with10\%) and peaks in April (15 species with 23\%) in the dry season for species such as Acrocarpus fraxinifolius, Anogeissus latifolia, Tectona grandis, and Terminalia paniculata. Leaf expansion begins in February (ten species with $15 \%$ ) and peaks in July (19 species with 29\%) for species including Dalbergia latifolia, Melia dubia, and Lannea coromandelica. Leaf senescence begins from September (two species with 3\%) and peaks in January (18 species with 28\%) for Haldina cordifolia, Careya arborea, and Tectona grandis (Table 1). In flowering phenology, out of 22 species, flower bud starts from February (two species with 3\%) and peaks in June (eight species with 12\%) for Schleichera oleosa and Lagerstroemia lanceolata. At the community level, opening of flowers begins in March (two species with 3\%) and peaks in May (five species with 7\%), and pollination of flowers starts from March (three species with 4\%) and peaks in July (five species with 7\%) for Albizia lebbeck and Terminalia tomentosa (Table 2).

Among 22 species, fruit bud starts from May (one species) and peaks in October (five species with 7\%) including Lannea coromandelica, Bombax malabaricum, and Butea monosperma. Unripened fruit starts from June (three species with 4\%) and peaks in November (nine species with 14\%). Ripened fruit starts from July (three species with 4\%) and peaks in December (ten species with 15\%) for Anogeissus latifolia, Bassia latifolia, 
Table 1 Leafing phenophases in different seasons of Bhadra wildlife sanctuary, India

\begin{tabular}{|c|c|c|c|c|c|}
\hline \multirow[t]{2}{*}{ Species } & \multirow[t]{2}{*}{ Seasons } & \multicolumn{4}{|c|}{ Leafing phenophases $^{\mathrm{a}}$} \\
\hline & & LF1 & LF2 & LF3 & LF4 \\
\hline \multirow[t]{3}{*}{ Acrocarpus fraxinifolius W. } & Rainy & 0 & 1 & 1 & 0 \\
\hline & Winter & 0 & 0 & 0 & 1 \\
\hline & Summer & 1 & 1 & 1 & 0 \\
\hline \multirow[t]{3}{*}{ Adina cordifolia (Roxb.)Ridsd. } & Rainy & 0 & 4 & 7 & 0 \\
\hline & Winter & 2 & 0 & 0 & 2 \\
\hline & Summer & 9 & 2 & 1 & 1 \\
\hline \multirow[t]{3}{*}{ Albizia odoratissima Benth. } & Rainy & 0 & 1 & 4 & 0 \\
\hline & Winter & 0 & 1 & 0 & 1 \\
\hline & Summer & 7 & 1 & 2 & 0 \\
\hline \multirow[t]{3}{*}{ Anogeissus latifolia Wall. } & Rainy & 4 & 3 & 7 & 0 \\
\hline & Winter & 2 & 0 & 1 & 3 \\
\hline & Summer & 10 & 4 & 2 & 1 \\
\hline \multirow[t]{3}{*}{ Bombax malabaricum DC. } & Rainy & 1 & 2 & 0 & 3 \\
\hline & Winter & 8 & 0 & 0 & 4 \\
\hline & Summer & 8 & 2 & 1 & 0 \\
\hline \multirow[t]{3}{*}{ Dalbergia latifolia Roxb. } & Rainy & 3 & 3 & 8 & 0 \\
\hline & Winter & 1 & 1 & 1 & 3 \\
\hline & Summer & 9 & 2 & 2 & 1 \\
\hline \multirow[t]{3}{*}{ Diospyros montana Roxb. } & Rainy & 0 & 0 & 1 & 0 \\
\hline & Winter & 0 & 1 & 0 & 1 \\
\hline & Summer & 0 & 0 & 1 & 0 \\
\hline \multirow[t]{3}{*}{ Ficus benghalensis L. } & Rainy & 0 & 1 & 2 & 1 \\
\hline & Winter & 0 & 1 & 0 & 1 \\
\hline & Summer & 0 & 1 & 3 & 0 \\
\hline \multirow[t]{3}{*}{ Ficus infectoria Roxb. } & Rainy & 0 & 1 & 1 & 0 \\
\hline & Winter & 0 & 1 & 0 & 1 \\
\hline & Summer & 0 & 1 & 1 & 0 \\
\hline \multirow[t]{3}{*}{ Ficus tsiela Roxb. } & Rainy & 0 & 1 & 1 & 0 \\
\hline & Winter & 0 & 1 & 0 & 1 \\
\hline & Summer & 0 & 1 & 0 & 0 \\
\hline \multirow[t]{3}{*}{ Kigelia pinnata DC. } & Rainy & 0 & 0 & 1 & 1 \\
\hline & Winter & 0 & 1 & 1 & 1 \\
\hline & Summer & 0 & 0 & 1 & 0 \\
\hline \multirow[t]{3}{*}{ Lagerstroemia lanceolata Wall. } & Rainy & 3 & 4 & 5 & 0 \\
\hline & Winter & 0 & 0 & 0 & 4 \\
\hline & Summer & 10 & 1 & 1 & 0 \\
\hline \multirow[t]{3}{*}{ Lannea coromandelica (Houtt.) Merr. } & Rainy & 1 & 4 & 5 & 5 \\
\hline & Winter & 7 & 0 & 0 & 10 \\
\hline & Summer & 10 & 5 & 4 & 0 \\
\hline \multirow[t]{3}{*}{ Melia dubia Hiern. } & Rainy & 0 & 2 & 2 & 0 \\
\hline & Winter & 0 & 0 & 1 & 1 \\
\hline & Summer & 0 & 1 & 3 & 0 \\
\hline
\end{tabular}

Table 1 Leafing phenophases in different seasons of Bhadra wildlife sanctuary, India (Continued)

\begin{tabular}{|c|c|c|c|c|c|}
\hline \multirow[t]{3}{*}{ Mitragyna parviflora Korth. } & Rainy & 0 & 5 & 5 & 0 \\
\hline & Winter & 0 & 1 & 2 & 2 \\
\hline & Summer & 8 & 3 & 3 & 1 \\
\hline \multirow[t]{3}{*}{ Pterocarpus marsupium Roxb. } & Rainy & 2 & 5 & 6 & 0 \\
\hline & Winter & 0 & 1 & 1 & 2 \\
\hline & Summer & 7 & 2 & 2 & 1 \\
\hline \multirow[t]{3}{*}{ Schleichera oleosa (Lour.) Oken. } & Rainy & 1 & 2 & 3 & 1 \\
\hline & Winter & 1 & 0 & 1 & 1 \\
\hline & Summer & 1 & 1 & 2 & 1 \\
\hline \multirow[t]{3}{*}{ Syzygium cumini (L.) Skeels. } & Rainy & 0 & 0 & 1 & 1 \\
\hline & Winter & 0 & 1 & 1 & 0 \\
\hline & Summer & 0 & 0 & 1 & 0 \\
\hline \multirow[t]{3}{*}{ Tectona grandis L.f. } & Rainy & 1 & 4 & 6 & 1 \\
\hline & Winter & 4 & 0 & 0 & 3 \\
\hline & Summer & 10 & 1 & 2 & 1 \\
\hline \multirow[t]{3}{*}{ Terminalia bellerica Roxb. } & Rainy & 0 & 1 & 2 & 0 \\
\hline & Winter & 0 & 0 & 0 & 1 \\
\hline & Summer & 1 & 0 & 1 & 1 \\
\hline \multirow[t]{3}{*}{ Terminalia paniculata Roth. } & Rainy & 0 & 2 & 4 & 0 \\
\hline & Winter & 3 & 0 & 0 & 2 \\
\hline & Summer & 10 & 1 & 2 & 1 \\
\hline \multirow[t]{3}{*}{ Terminalia tomentosa W. \& A. } & Rainy & 1 & 2 & 4 & 1 \\
\hline & Winter & 0 & 0 & 0 & 3 \\
\hline & Summer & 10 & 3 & 3 & 0 \\
\hline
\end{tabular}

aLeafing phenophases: LF1, leafless; LF2, leaf initiation; LF3, immature leaf; and LF4, leaf senescence.

and Cassine glauca. Fruit maturation took place for 4 months' duration for Lagerstroemia lanceolata and 5 months' duration for Tectona grandis. Fruit senescence starts from August (five species with 7\%) and peaks in February (nine species with 14\%) during the monsoon to post-monsoon period for Haldina cordifolia, Anogeissus latifolia, Terminalia paniculata, Terminalia tomentosa, and Ziziphus xylopyrus (Table 3).

\section{Factors influencing phenology}

Leaf initiation is not significant to rainfall during current months and had a strong negative significance during a 3-month lag period $\left(r_{s}=-0.70, P<0.0003\right)$ (Figure 2). Maximum temperature had a positive influence $\left(\mathrm{r}_{\mathrm{s}}=\right.$ $0.40, P<0.03)$ during current months and a 1-month lag period $\left(\mathrm{r}_{\mathrm{s}}=0.41, P<0.05\right)$. Minimum temperature is not significant during current months and had a significant strong negative influence during a 3-month lag period $\left(\mathrm{r}_{\mathrm{s}}=-0.81, P<0.0000006\right)$. Leaf expansion and rainfall had a strong negative significance during a 3-month lag 
Table 2 Flowering phenophases in different seasons of Bhadra wildlife sanctuary, India

\begin{tabular}{|c|c|c|c|c|}
\hline \multirow[t]{2}{*}{ Species } & \multirow[t]{2}{*}{ Seasons } & \multicolumn{3}{|c|}{$\begin{array}{c}\text { Flowering } \\
\text { phenophases }^{\mathrm{a}}\end{array}$} \\
\hline & & FL1 & FL2 & FL3 \\
\hline \multirow[t]{3}{*}{ Acrocarpus fraxinifolius W. } & Rainy & 0 & 0 & 0 \\
\hline & Winter & 0 & 0 & 0 \\
\hline & Summer & 0 & 0 & 0 \\
\hline \multirow[t]{3}{*}{ Adina cordifolia (Roxb.)Ridsd. } & Rainy & 1 & 1 & 1 \\
\hline & Winter & 0 & 0 & 0 \\
\hline & Summer & 0 & 0 & 0 \\
\hline \multirow[t]{3}{*}{ Albizia odoratissima Benth. } & Rainy & 0 & 1 & 0 \\
\hline & Winter & 0 & 0 & 0 \\
\hline & Summer & 3 & 1 & 1 \\
\hline \multirow[t]{3}{*}{ Anogeissus latifolia Wall. } & Rainy & 2 & 2 & 1 \\
\hline & Winter & 0 & 0 & 0 \\
\hline & Summer & 0 & 0 & 0 \\
\hline \multirow[t]{3}{*}{ Bombax malabaricum DC. } & Rainy & 0 & 0 & 0 \\
\hline & Winter & 1 & 1 & 1 \\
\hline & Summer & 1 & 1 & 0 \\
\hline \multirow[t]{3}{*}{ Dalbergia latifolia Roxb. } & Rainy & 1 & 0 & 0 \\
\hline & Winter & 0 & 0 & 0 \\
\hline & Summer & 0 & 0 & 0 \\
\hline \multirow[t]{3}{*}{ Diospyros montana Roxb. } & Rainy & 0 & 0 & 0 \\
\hline & Winter & 0 & 0 & 0 \\
\hline & Summer & 0 & 0 & 0 \\
\hline \multirow[t]{3}{*}{ Ficus benghalensis $\mathrm{L}$. } & Rainy & 0 & 0 & 0 \\
\hline & Winter & 0 & 0 & 0 \\
\hline & Summer & 1 & 0 & 0 \\
\hline \multirow[t]{3}{*}{ Ficus infectoria Roxb. } & Rainy & 0 & 0 & 0 \\
\hline & Winter & 1 & 0 & 0 \\
\hline & Summer & 0 & 0 & 0 \\
\hline \multirow[t]{3}{*}{ Ficus tsiela Roxb. } & Rainy & 1 & 1 & 0 \\
\hline & Winter & 1 & 0 & 0 \\
\hline & Summer & 0 & 0 & 0 \\
\hline \multirow[t]{3}{*}{ Kigelia pinnata DC. } & Rainy & 0 & 0 & 0 \\
\hline & Winter & 1 & 1 & 1 \\
\hline & Summer & 1 & 1 & 1 \\
\hline \multirow[t]{3}{*}{ Lagerstroemia lanceolata Wall. } & Rainy & 1 & 1 & 1 \\
\hline & Winter & 0 & 0 & 0 \\
\hline & Summer & 0 & 0 & 0 \\
\hline \multirow[t]{3}{*}{ Lannea coromandelica (Houtt.) Merr. } & Rainy & 1 & 1 & 0 \\
\hline & Winter & 2 & 0 & 0 \\
\hline & Summer & 1 & 1 & 1 \\
\hline \multirow[t]{3}{*}{ Melia dubia Hiern. } & Rainy & 0 & 0 & 0 \\
\hline & Winter & 1 & 0 & 0 \\
\hline & Summer & 0 & 0 & 0 \\
\hline
\end{tabular}

Table 2 Flowering phenophases in different seasons of Bhadra wildlife sanctuary, India (Continued)

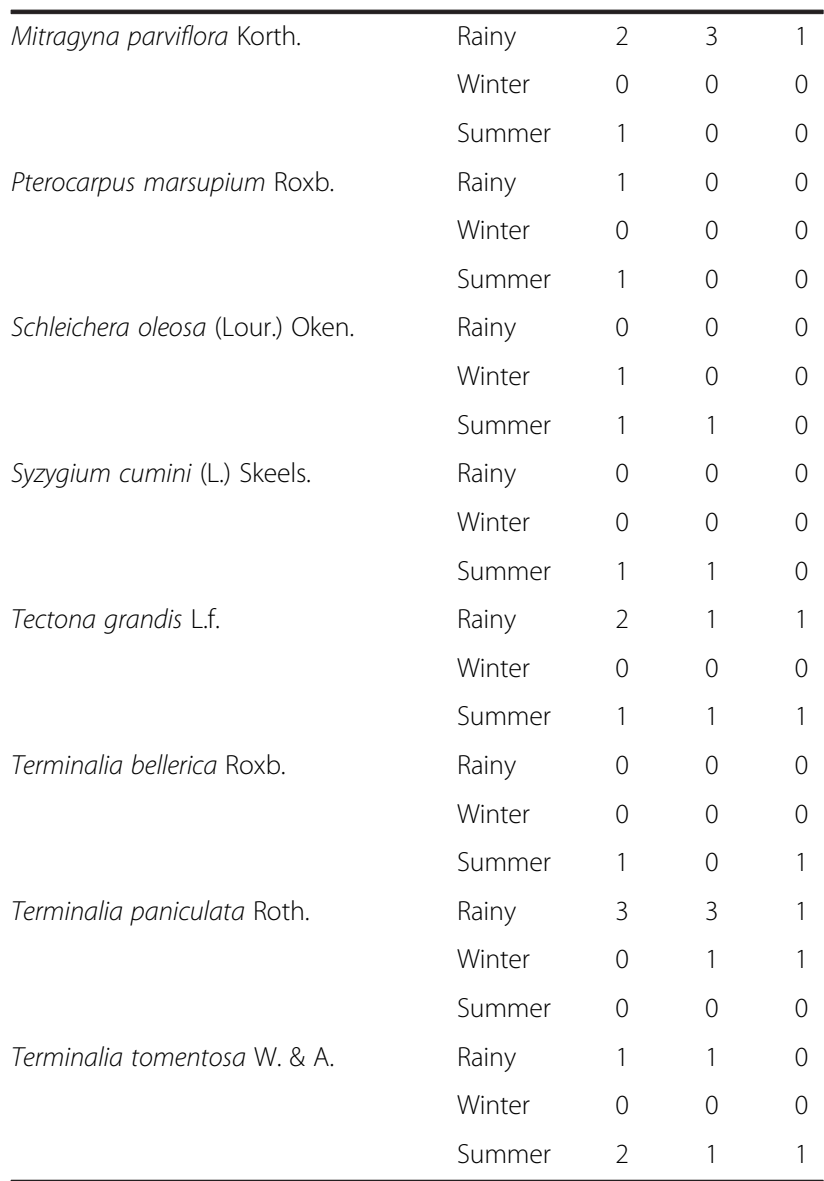

${ }^{a}$ Flowering phenophases: FL1, flower initiation/flower bud; FL2, opening flower; FL3, pollinating flower.

period $\left(\mathrm{r}_{\mathrm{s}}=-0.68, P<0.0005\right)$. Maximum temperature had a positive influence during current months $\left(r_{\mathrm{s}}=\right.$ $0.45, P<0.02)$, and 1 -month $\left(\mathrm{r}_{\mathrm{s}}=0.58, P<0.003\right)$ and 2 -month $\left(\mathrm{r}_{\mathrm{s}}=0.47, P<0.02\right)$ lag periods (Figure 3$)$. However minimum temperature is negatively significant during a 2-month lag period $\left(\mathrm{r}_{\mathrm{s}}=-0.51, P<0.01\right)$ and had a strong negative influence during a 3-month lag period $\left(\mathrm{r}_{\mathrm{s}}=-0.74, P<0.0001\right)$. Leaf senescence and rainfall had a strong negative influence during current months $\left(\mathrm{r}_{\mathrm{s}}=-0.73, P<0.00005\right)$ with a negative influence during a 1 -month lag period $\left(\mathrm{r}_{\mathrm{s}}=-0.44, P<0.03\right)$. Maximum temperature during a 1 -month $\left(r_{\mathrm{s}}=-0.47, P<0.02\right)$, 2month $\left(\mathrm{r}_{\mathrm{s}}=-0.53, P<0.009\right)$, and 3-month $\left(\mathrm{r}_{\mathrm{s}}=-0.55\right.$, $P<0.009)$ lag periods had a significant negative influence. Minimum temperature $\left(\mathrm{r}_{\mathrm{s}}=-0.68, P<0.0001\right)$ is negatively significant during current months (Figure 4 ).

Flower initiation had a negative influence during 2-month $\left(\mathrm{r}_{\mathrm{s}}=-0.43, P<0.04\right)$ and 3-month $\left(\mathrm{r}_{\mathrm{s}}=-0.71\right.$, $P<0.0002$ ) lag periods (Figure 5). However maximum temperature had a positive influence during current months $\left(r_{s}=0.41, P<0.04\right)$, and 1-month $\left(r_{s}=0.57\right.$, 
Table 3 Fruiting phenophases in different seasons of Bhadra wildlife sanctuary, India

\begin{tabular}{|c|c|c|c|c|c|}
\hline \multirow[t]{2}{*}{ Species } & \multirow[t]{2}{*}{ Seasons } & \multicolumn{4}{|c|}{ Fruiting phenophases $^{a}$} \\
\hline & & FR1 & FR2 & FR3 & FR4 \\
\hline \multirow[t]{3}{*}{ Acrocarpus fraxinifolius W. } & Rainy & 0 & 0 & 0 & 0 \\
\hline & Winter & 0 & 0 & 0 & 0 \\
\hline & Summer & 0 & 0 & 0 & 0 \\
\hline \multirow[t]{3}{*}{ Adina cordifolia (Roxb.)Ridsd. } & Rainy & 2 & 1 & 2 & 1 \\
\hline & Winter & 1 & 1 & 5 & 1 \\
\hline & Summer & 0 & 0 & 1 & 3 \\
\hline \multirow[t]{3}{*}{ Albizia odoratissima Benth. } & Rainy & 0 & 0 & 0 & 0 \\
\hline & Winter & 0 & 1 & 0 & 0 \\
\hline & Summer & 0 & 0 & 1 & 1 \\
\hline \multirow[t]{3}{*}{ Anogeissus latifolia Wall. } & Rainy & 1 & 2 & 1 & 0 \\
\hline & Winter & 1 & 1 & 5 & 1 \\
\hline & Summer & 0 & 0 & 1 & 5 \\
\hline \multirow[t]{3}{*}{ Bombaxma labaricum DC. } & Rainy & 0 & 1 & 1 & 0 \\
\hline & Winter & 0 & 0 & 0 & 0 \\
\hline & Summer & 0 & 1 & 0 & 1 \\
\hline \multirow[t]{3}{*}{ Dalbergia latifolia Roxb. } & Rainy & 0 & 0 & 1 & 0 \\
\hline & Winter & 0 & 0 & 0 & 0 \\
\hline & Summer & 0 & 0 & 0 & 0 \\
\hline \multirow[t]{3}{*}{ Diospyros montana Roxb. } & Rainy & 0 & 0 & 0 & 0 \\
\hline & Winter & 0 & 0 & 0 & 0 \\
\hline & Summer & 0 & 0 & 0 & 0 \\
\hline \multirow[t]{3}{*}{ Ficus benghalensis L. } & Rainy & 0 & 1 & 1 & 1 \\
\hline & Winter & 1 & 1 & 0 & 0 \\
\hline & Summer & 0 & 1 & 1 & 1 \\
\hline \multirow[t]{3}{*}{ Ficus infectoria Roxb. } & Rainy & 0 & 0 & 0 & 0 \\
\hline & Winter & 0 & 0 & 0 & 0 \\
\hline & Summer & 1 & 1 & 0 & 0 \\
\hline \multirow[t]{3}{*}{ Ficus tsiela Roxb. } & Rainy & 0 & 0 & 0 & 0 \\
\hline & Winter & 1 & 0 & 0 & 0 \\
\hline & Summer & 0 & 0 & 0 & 0 \\
\hline \multirow[t]{3}{*}{ Kigelia pinnata DC. } & Rainy & 0 & 0 & 0 & 0 \\
\hline & Winter & 0 & 0 & 0 & 0 \\
\hline & Summer & 0 & 0 & 0 & 0 \\
\hline \multirow[t]{3}{*}{ Lagerstroemia lanceolata Wall. } & Rainy & 0 & 0 & 0 & 0 \\
\hline & Winter & 0 & 0 & 0 & 0 \\
\hline & Summer & 0 & 0 & 1 & 1 \\
\hline \multirow[t]{3}{*}{ Lannea coromandelica (Houtt.) Merr. } & Rainy & 0 & 0 & 0 & 0 \\
\hline & Winter & 0 & 0 & 0 & 0 \\
\hline & Summer & 2 & 2 & 4 & 5 \\
\hline \multirow[t]{3}{*}{ Melia dubia Hiern. } & Rainy & 0 & 0 & 0 & 0 \\
\hline & Winter & 0 & 0 & 0 & 0 \\
\hline & Summer & 0 & 1 & 1 & 0 \\
\hline
\end{tabular}

Table 3 Fruiting phenophases in different seasons of Bhadra wildlife sanctuary, India (Continued)

\begin{tabular}{|c|c|c|c|c|c|}
\hline \multirow[t]{3}{*}{ Mitragyna parviflora Korth. } & Rainy & 1 & 2 & 5 & 1 \\
\hline & Winter & 0 & 1 & 10 & 0 \\
\hline & Summer & 0 & 0 & 8 & 7 \\
\hline \multirow[t]{3}{*}{ Pterocarpus marsupium Roxb. } & Rainy & 0 & 0 & 0 & 0 \\
\hline & Winter & 0 & 0 & 0 & 0 \\
\hline & Summer & 0 & 0 & 0 & 0 \\
\hline \multirow[t]{3}{*}{ Schleichera oleosa (Lour.) Oken. } & Rainy & 0 & 0 & 1 & 1 \\
\hline & Winter & 0 & 0 & 0 & 0 \\
\hline & Summer & 0 & 1 & 0 & 1 \\
\hline \multirow[t]{3}{*}{ Syzygium cumini (L.) Skeels. } & Rainy & 0 & 0 & 0 & 0 \\
\hline & Winter & 0 & 0 & 0 & 0 \\
\hline & Summer & 0 & 0 & 0 & 0 \\
\hline \multirow[t]{3}{*}{ Tectona grandis L.f. } & Rainy & 1 & 2 & 1 & 1 \\
\hline & Winter & 0 & 1 & 1 & 6 \\
\hline & Summer & 0 & 0 & 0 & 1 \\
\hline \multirow[t]{3}{*}{ Terminalia bellerica Roxb. } & Rainy & 0 & 0 & 0 & 0 \\
\hline & Winter & 0 & 1 & 0 & 0 \\
\hline & Summer & 0 & 1 & 0 & 0 \\
\hline \multirow[t]{3}{*}{ Terminalia paniculata Roth. } & Rainy & 1 & 1 & 0 & 0 \\
\hline & Winter & 2 & 2 & 4 & 5 \\
\hline & Summer & 0 & 0 & 0 & 2 \\
\hline \multirow[t]{3}{*}{ Terminalia tomentosa W. \& A. } & Rainy & 2 & 2 & 1 & 1 \\
\hline & Winter & 0 & 1 & 2 & 3 \\
\hline & Summer & 0 & 0 & 1 & 2 \\
\hline
\end{tabular}

${ }^{a}$ Fruiting phenophases: $F R 1$, fruit initiation; FR2, unripened fruit; FR3, ripened fruit; FR4, fruit senescence.

$P<0.004)$ and 2-month $\left(\mathrm{r}_{\mathrm{s}}=0.68, P<0.0004\right)$ lag periods. Minimum temperature had an influence during 2-month $\left(\mathrm{r}_{\mathrm{s}}=-0.51, P<0.01\right)$ and 3-month $\left(\mathrm{r}_{\mathrm{s}}=-0.61\right.$, $P<0.002)$ lag periods. Open flowering phenology and rainfall had a negative influence during 2-month $\left(\mathrm{r}_{\mathrm{s}}=-0.45, P<0.03\right)$ and 3-month $\left(\mathrm{r}_{\mathrm{s}}=-0.52, P<0.01\right)$ lag periods. Maximum temperature had a positive significance during 2-month $\left(\mathrm{r}_{\mathrm{s}}=0.52, P<0.009\right)$ and 3month $\left(r_{s}=0.67, P<0.0005\right)$ lag periods, and minimum temperature had a negative significance during 2-month $\left(\mathrm{r}_{\mathrm{s}}=-0.48, P<0.02\right)$ and 3-month $\left(\mathrm{r}_{\mathrm{s}}=-0.54, P<0.01\right)$ lag periods. Pollinating flowering phenology and maximum temperature had a positive influence during 1month $\left(\mathrm{r}_{\mathrm{s}}=0.41, P<0.05\right)$, 2-month $\left(\mathrm{r}_{\mathrm{s}}=0.52, P<0.01\right)$, and 3-month $\left(\mathrm{r}_{\mathrm{s}}=0.49, P<0.02\right)$ lag periods (Figure 6).

Fruit initiation had no significant influence with rainfall and maximum and minimum temperature during current months and 1- to 3-month lag periods. Fruit maturing phase with rainfall had a positive influence during a 3-month $\left(\mathrm{r}_{\mathrm{s}}=0.51, P<0.01\right)$ lag period. Maximum temperature had a negative influence during 


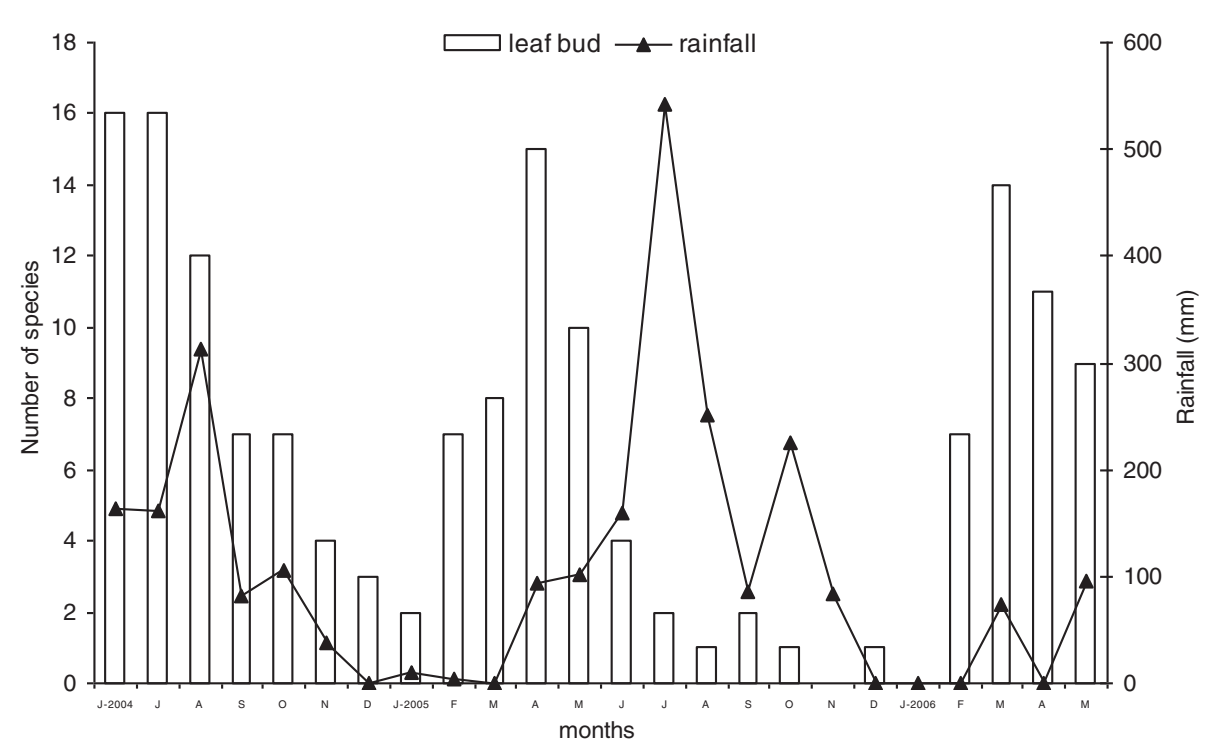

Figure 2 Leaf initiation and rainfall in canopy trees of Bhadra wildlife sanctuary.

1-month $\left(\mathrm{r}_{\mathrm{s}}=-0.43, P<0.03\right)$ and 2 -month $\left(\mathrm{r}_{\mathrm{s}}=-0.69\right.$, $P<0.0003)$ lag periods. Ripened fruit stage had a negative influence with rainfall during current months $\left(\mathrm{r}_{\mathrm{s}}=-0.54, P<0.006\right)$ and a 1 -month $\left(\mathrm{r}_{\mathrm{s}}=-0.40, P<0.05\right)$ lag period, and minimum temperature had a negative influence during a 1 -month $\left(\mathrm{r}_{\mathrm{s}}=-0.45, P<0.03\right)$ lag period. Fruit fall phenology and rainfall had a significant negative influence during current months $\left(\mathrm{r}_{\mathrm{s}}=-0.59, P<0.002\right)$, 1-month $\quad\left(\mathrm{r}_{\mathrm{s}}=-0.58, \quad P<0.003\right), \quad 2$-month $\quad\left(\mathrm{r}_{\mathrm{s}}=-0.64\right.$, $P<0.001)$, and 3 -month $\left(\mathrm{r}_{\mathrm{s}}=-0.60, P<0.003\right)$ lag periods. Maximum temperature had a positive influence during current months $\left(\mathrm{r}_{\mathrm{s}}=0.44, \quad P<0.02\right)$, and minimum temperature had a significant negative influence during current months $\left(\mathrm{r}_{\mathrm{s}}=-0.47, \quad P<0.01\right)$, and 1-month $\left(\mathrm{r}_{\mathrm{s}}=-0.62, P<0.001\right)$ and2-month $\left(\mathrm{r}_{\mathrm{s}}=-0.54, P<0.008\right)$ lag periods (Figure 7).

\section{Seasonality among various phenophases}

Seasonality of various leafing phenophases is strongly pronounced. Rayleigh's Z values are highly significant. The leafing pattern is indicated by the mean angle. Most trees are leafless in the middle of February as indicated

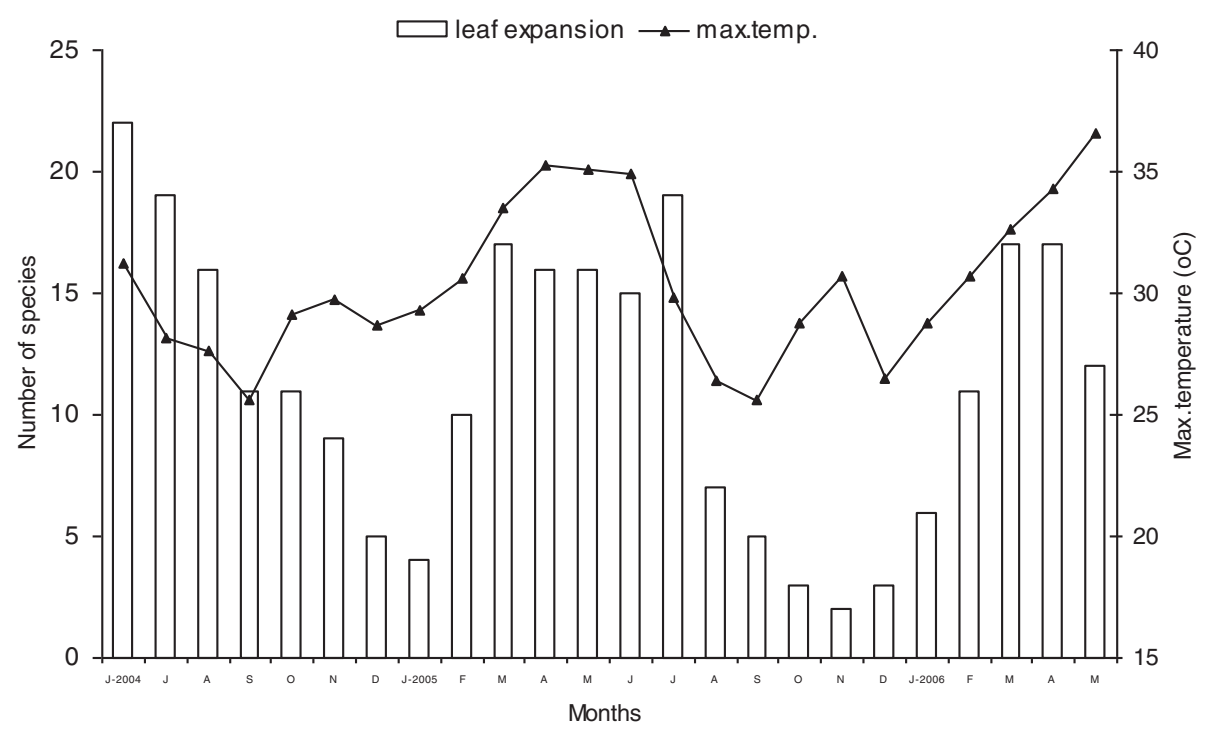

Figure 3 Leaf expansion and maximum temperature in canopy trees of Bhadra wildlife sanctuary. 


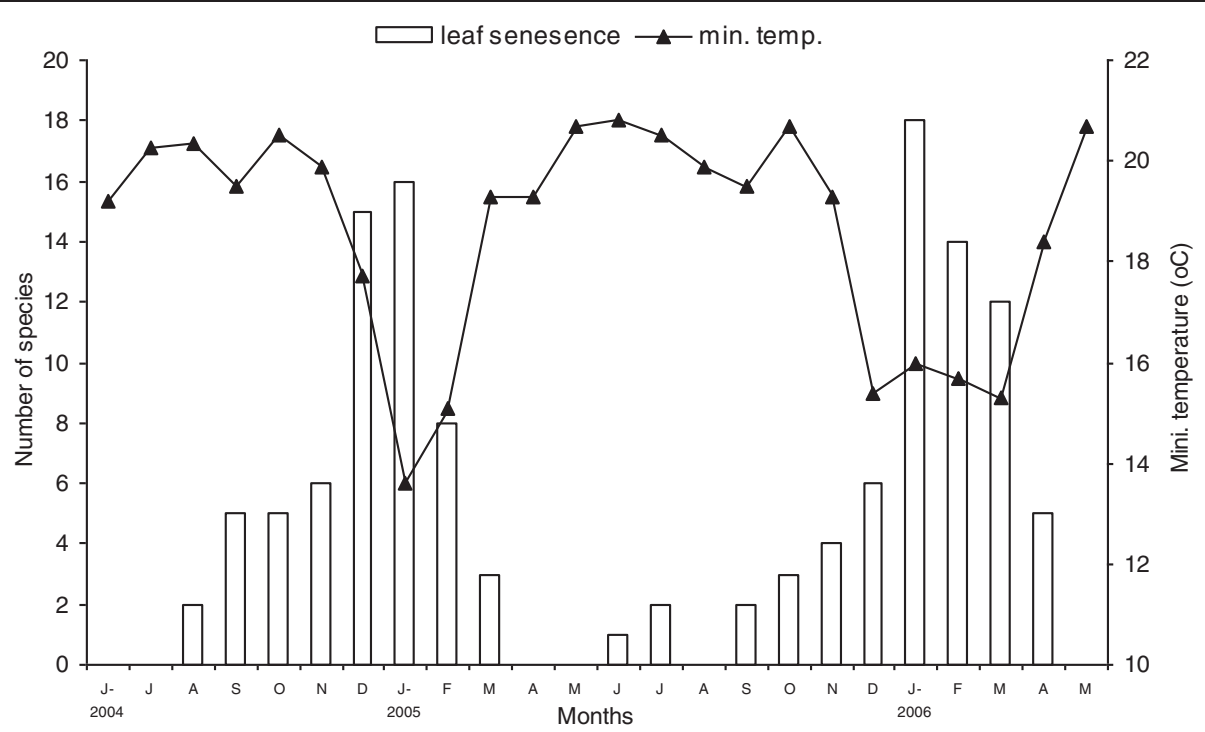

Figure 4 Leaf senescence and minimum temperature in canopy trees of Bhadra wildlife sanctuary.

by the mean angle (72.25). Leaf initiation starts in the middle of May (136.0), leaf expansion at the end of May (146.0), and senescence in the middle of January (15.95). The strength of seasonality measured by the vector $r$ indicates that the leaf senescence (0.58) event has strong seasonality, followed by leaf initiation (0.37) and leaf expansion (0.31) (Table 4). Seasonality of various flowering phenophases is also strongly pronounced. Rayleigh's Z values are significant as indicated by the mean angle. In dry deciduous forests most tree species initiate flower bud in the middle of May (136.4), and open and become pollinated flowers during late May
(144.07 and 143.16, respectively). The strength of seasonality measured by the vector $r$ indicates that open flowers $(r=0.37)$ have strong seasonality, followed by flower initiation $(r=0.29)$ and pollinated flowers $(r=0.27)$ (Table 5). Seasonality of various fruiting phenophases is notable. Rayleigh's Z is highly significant, and the significance of the fruiting pattern is indicated by the mean angle. Fruit initiation happened during the middle of February (50.34). Immature fruits were observed during the middle of November (318.54), fruits ripened at the beginning of February (31.70), and fruit fall occurred in the middle of March (74.84). The

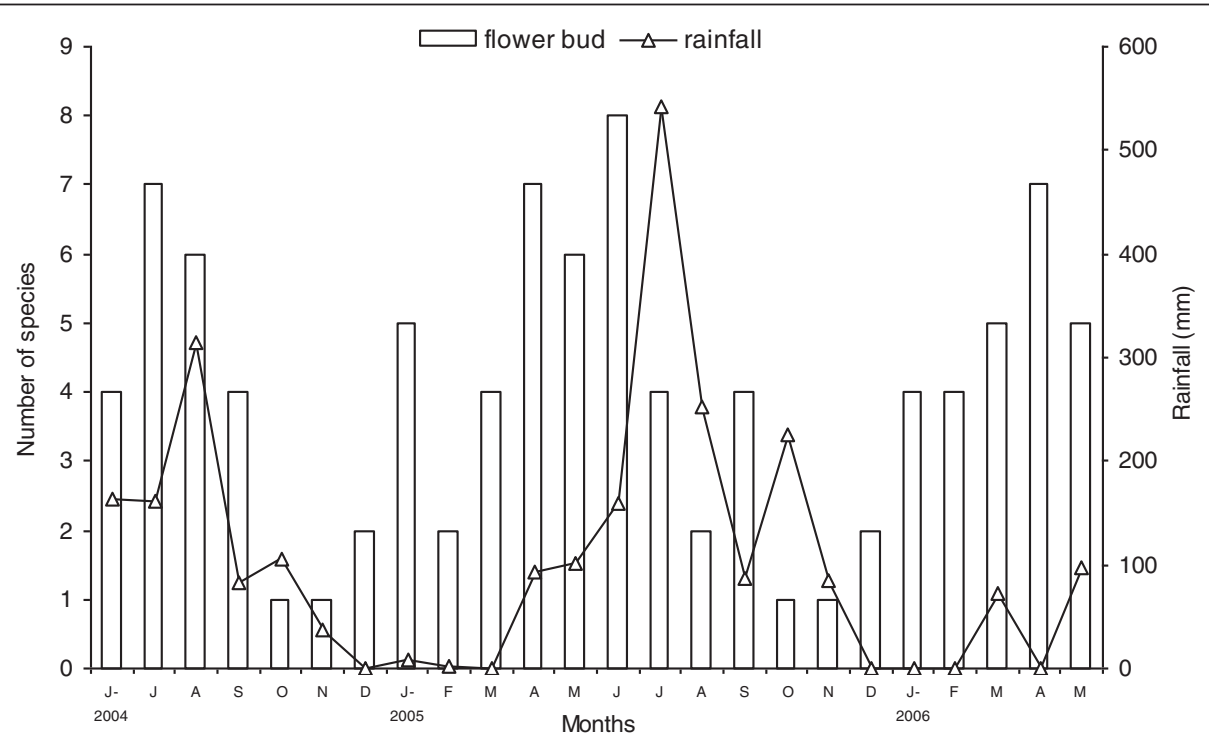

Figure 5 Flower bud and rainfall in canopy trees of Bhadra wildlife sanctuary. 


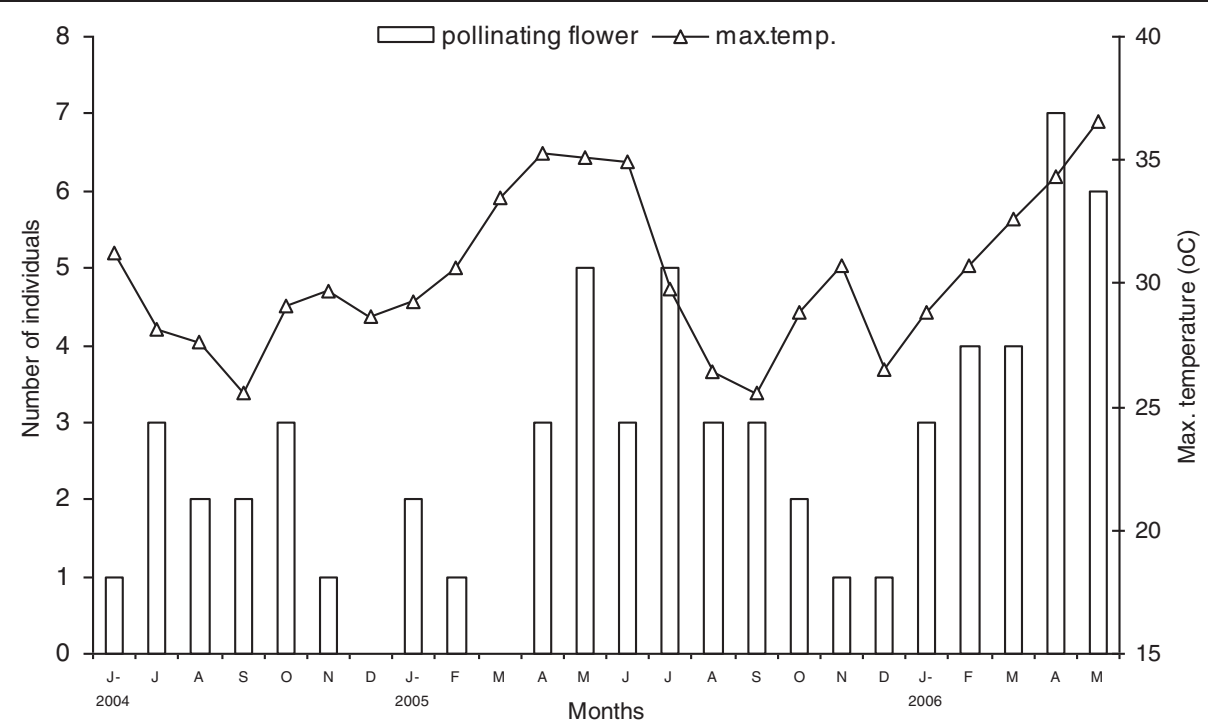

Figure 6 Pollinating flower and maximum temperature in canopy trees of Bhadra wildlife sanctuary.

strength of seasonality measured by vector $\mathrm{r}$ indicated that fruit fall $(\mathrm{r}=0.34)$ has strong seasonality, and immature and ripened fruit $(r=0.22)$ showed similar seasonality, followed by fruit initiation $(r=0.03)$ (Table 6).

\section{Discussion}

Trees of Bhadra wildlife sanctuary respond to leaf flush during the dry season (Nanda 2009), and dry season flushing was more pronounced in the present study and elsewhere (Frankie et al. 1974; Van Schaik 1986; Bhat 1992; Murali and Sukumar 1993; Whitmore 1996). In tropical trees initiation of leaf flush (leaf bud), marking the termination of deciduousness duration, has been reported to be triggered by several factors such as increasing day length and/or temperature, significant amounts of first rain, and photoperiod (Rivera et al. 2002; Singh and Kushwaha, 2005; Daubenmire 1972). Moisture appears to be a major determinant of the timing of leaf flush in the dry tropical forest of Ghana (Lieberman 1982).

Most deciduous species of dry monsoon forests in Thailand and India form new leaves 1 to 2 months before the first monsoon rains, during the hottest and driest part of the year around the spring equinox (Elliot

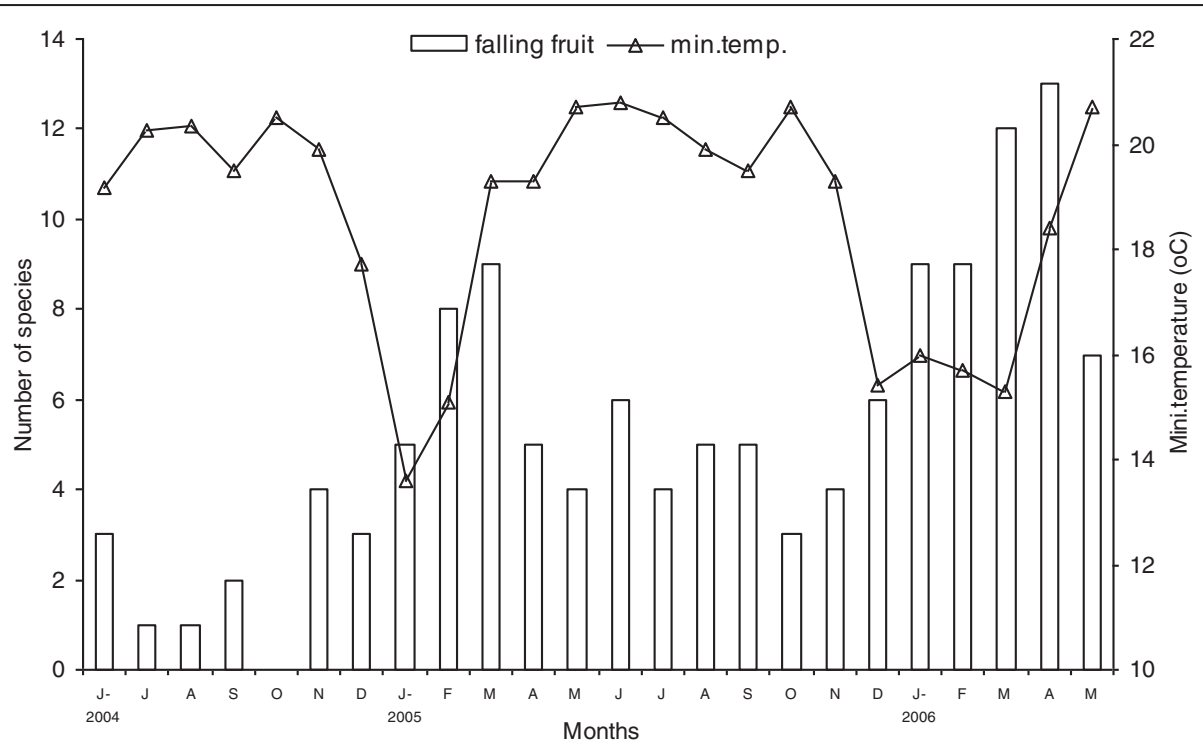

Figure 7 Falling fruit and minimum temperature in canopy trees of Bhadra wildlife sanctuary. 
Table 4 Leafing phenology seasonality in dry deciduous forest ofBhadra wildlife sanctuary, India

\begin{tabular}{lcccc}
\hline Parameters & Leafless & $\begin{array}{c}\text { Leaf } \\
\text { initiation }\end{array}$ & $\begin{array}{c}\text { Leaf } \\
\text { expansion }\end{array}$ & $\begin{array}{c}\text { Leaf } \\
\text { senescence }\end{array}$ \\
\hline Mean angle & 72.25 & 136.0 & 146.0 & 15.95 \\
Mean vector $r$ & 0.616 & 0.37 & 0.31 & 0.58 \\
Angular SD & 56.40 & 80.45 & 87.67 & 59.04 \\
Rayleigh's Z & 35.28 & 22.13 & 26.25 & 43.91 \\
$P$ value & $<0.000^{\mathrm{a}}$ & $<0.000^{\mathrm{a}}$ & $<0.000^{\mathrm{a}}$ & $<0.000^{\mathrm{a}}$ \\
\hline
\end{tabular}

${ }^{\mathrm{a}}$ Significant at $<0.05 . \mathrm{SD}$, standard deviation.

et al. 2006). Leaf expansion differs between species; for example 3 months in Pterocarpus marsupium and 4 months in Lagerstroemia lanceolata (Nanda et al. 2013a).

Bolivian dry forest canopy species lose their leaves during the dry season (Justiniano and Fredericksen 2000). Several studies suggest that low temperatures (for example daily mean temperature below a $8^{\circ} \mathrm{C}$ threshold; Estrella and Menzel 2006) and short days (Koike 1990, with a 13-hour photoperiod threshold by $63^{\circ} \mathrm{N}$ latitude in Keskitalo et al. 2005) are among the main factors involved in triggering leaf senescence in deciduous trees. Borchert (1983) suggested that the leaffall during the dry season was directly influenced by the decline in soil moisture and increasing water stress conditions. No soil moisture data was available from the present study to justify other studies.

Our observations also show that leaf senescence peaks during the low mean minimum temperature period, and the absence of leaf or no leaf phase begins from January in one or the other species with a peak in February and March. The results are also in conformity with Singh and Singh (1992) who reported that initiation of leaf fall coincides with the onset of the post-monsoon low temperature dry period and can be a mechanism maintaining turgidity of shoots.

A few species produced flowers during the leafless phase including Lannea coromandelica, Bombax malabaricum, Butea monosperma, and Melia dubia (Nanda et al. 2010). In the Mudumalai dry forest, species included Bombax ceiba, Erythrina indica, Butea monosperma, and Radermachera xylocarpa (Murali and Sukumar 1994).

Table 5 Flowering phenology seasonality in dry deciduous forest ofBhadra wildlife sanctuary, India

\begin{tabular}{lccc}
\hline Parameters & Flower initiation & Open flowers & Pollinating flowers \\
\hline Mean angle & 136.4 & 144.07 & 143.16 \\
Mean vector $r$ & 0.29 & 0.37 & 0.27 \\
Angular SD & 89.42 & 79.78 & 92.35 \\
Rayleigh's Z & 8.40 & 8.71 & 4.38 \\
$P$ value & $<0.000^{\mathrm{a}}$ & $<0.000^{\mathrm{a}}$ & $<0.008^{\mathrm{a}}$ \\
\hline
\end{tabular}

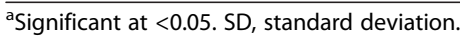

Table 6 Fruiting phenology seasonality in dry deciduous forest ofBhadra wildlife sanctuary, India

\begin{tabular}{lcccc}
\hline Parameters & $\begin{array}{c}\text { Fruit } \\
\text { initiation }\end{array}$ & $\begin{array}{c}\text { Fruit } \\
\text { immaturity }\end{array}$ & $\begin{array}{c}\text { Fruit } \\
\text { maturity }\end{array}$ & $\begin{array}{c}\text { Fruit } \\
\text { fall }\end{array}$ \\
\hline Mean angle & 50.34 & 318.54 & 31.70 & 74.84 \\
Mean vector $r$ & 0.03 & 0.22 & 0.22 & 0.34 \\
Angular SD & 149.88 & 99.52 & 98.43 & 83.65 \\
Rayleigh's Z & 0.073 & 4.60 & 7.36 & 15.18 \\
$P$ value & $<0.931$ & $<0.01^{\text {a }}$ & $<0.001^{\text {a }}$ & $<0.000^{\text {a }}$
\end{tabular}

${ }^{a}$ Significant at $<0.05$. SD, standard deviation.

However at the community level flower initiation begins in January and peaks from March to April (Nanda et al. 2010). The leafless phase favours wind pollination as well as floral display to attract pollinators (Singh and Singh 1992). Nanda et al. (2011, 2013b) reported synchronization of flowering and leaf flushing in a few species of the present study, namely Schleichera oleosa and Lagerstroemia lanceolata, which seem to be related to moisture, temperature, and day length, and this observation, is in conformity with those of Boojh and Ramakrishnan (1981) and Murali and Sukumar (1994). In the seasonal tropical rain forests in peninsular Malaysia and Borneo, a drop of $2^{\circ} \mathrm{C}$ in daily temperature for several days has been speculated to be a proximal cue for general flowering (Ashton et al. 1988; Sakai et al. 1999; Yasuda et al. 1999). Phenological studies in seasonal tropical forest ecosystems have indicated rainfall seasonality as being the major abiotic factor controlling the timing, intensity, and duration of flowering and fruiting periodicities (Singh and Singh 1992; Newstrom et al. 1994; Sun et al. 1996; Borchert et al. 2004). Lieberman (1982) predicted that in some species, the time-lag between two events reflects partitioning of resources for various physiological activities. Solar irradiance and rainfall are often considered as the main seasonal variables influencing the flowering phenology of tropical plants (Wright and Van Schaik 1994; Wright 1996).

Water storage in tree trunks may enable maintenance of high stem water potential and flowering during the dry season (Schongart et al. 2002). Burger (1974) reported in Ethiopian dry forests that the flowering response was primarily in the wet season and in the dry season in evergreen montane forests.

Fruit initiation happens with reduction in minimum temperature or with the advent of a warm period. In the present study, the initiation of the fruit bud happened in certain species with leaf expansion including Bassia latifolia, Semecarpus anacardium, Lagerstroemia lanceolata, and Tectona grandis. For fruits to mature, trees require temporally concentrated rainfall during the preceding months. A similar trend was also observed in the dry forest of Australia (Bach 2002), in other dry forests of 
India (Murali and Sukumar 1994; Krishnan 2002), and tropical montane forests in Rwanda (Sun et al. 1996). In Bhadra wildlife sanctuary ripening of fruit began in March and increased with the onset of the wet season from August to September. Ripened fruiting peaks have been reported to occur in the wet season (Foster 1982; Heideman 1989; Chapman et al. 1999). Fleshy-fruited species produced fruits preferentially during the wet season in the dry tropical forest of Ghana (Lieberman 1982). Peak activity of fruiting occurred in the dry season (Chapman et al. 2005), and pre-monsoon fruit ripening occurred in the dry period or close to rainfall to gain postdispersal success (Rathcke and Lacey 1985), enhance dispersal (Prasad and Sharatchandra 1984; Sundrapandian et al. 2005), and to avoid pathogen infection (Augspurger, 1983).

Previous seasonality studies on phenology from India (Singh and Singh 1992; Murali and Sukumar 1993, 1994; Bhat 1992; Prasad and Hegde 1986) mention the seasons of leafing, flowering, and fruiting at the community level. From this study, by using Rayleigh's $Z$ test, various phenophases were expressed quantitatively and qualitatively in the dry forests of Bhadra wildlife sanctuary, which were shown to be significantly seasonal with a seasonal climatic factor. Despite the lack of seasonality in climate, attempts to quantify the seasonality of a phenological stage at the community level have shown significant seasonality (Morellato et al. 2000). With a strong seasonality in rainfall, the leaf phenophases also showed significant seasonality (Suresh and Sukumar 2011).

\section{Conclusion}

Phenology of leaf initiation and expansion is concentrated during the pre-monsoon and leaf senescence postmonsoon. Flowering phenology peaked during summer, fruit bud and ripening during the monsoon, and fruit fall post-monsoon. Seasonality studies reveal that leaf initiation and flower budding happen simultaneously. The phenological patterns exhibited by tropical dry forest species seem to be related to rainfall and temperature distribution. Tropical forests have a great diversity in phenological patterns within and among forests resulting from many short-term variations in phenology, which may be associated with alterations in rainfall, temperature, relative humidity, hours of sunshine, and other unnoticed factors. This short-term duration data helps to understand the environmental perturbations on phenology; however, longterm studies that include more environmental factors are needed to understand their stability.

\section{Competing interests}

The authors declare that they have no competing interests.

\section{Authors' contributions}

AN designed the study, managed and analyzed the data, and wrote the manuscript. HSS assisted in making the calculations and analysis, and helped in writing the manuscript. AN HSS and YLK discussed the different topics. All authors read and approved the final manuscript.

\section{Acknowledgements}

The authors thank the Karnataka Forest Department for permission to work in Bhadra wildlife sanctuary, and the Bhadra River Project meteorological station for data on rainfall and temperature.

\section{Author details}

${ }^{1}$ Department of Postgraduate Studies and Research in Applied Botany, Bioscience Complex, Jnana Sahyadri, Kuvempu University, Shankaraghatta, Shimoga 577 451, Karnataka, India. 'Biodiversity Education and Research Laboratory, Environmental Study Centre, Santhekaduru, Shimoga 577222, Karnataka, India. ${ }^{3}$ Centre for Ecological Sciences, Indian Institute of Science, Bangalore 560012, Karnataka, India.

Received: 15 August 2013 Accepted: 12 December 2013

Published: 10 January 2014

\section{References}

Aide TM (1993) Patterns of leaf development and herbivory in a tropical understory community. Ecology 74:455-466

Appanah S (1985) General flowering in the climax rain forests of south-east Asia. J Trop Ecol 1:225-240

Ashton PS, Givnish TJ, Appanah S (1988) Staggered flowering in the Dipterocarpaceae: new insights into floral induction and the evolution of mast fruiting in the aseasonal tropics. Am Nat 132:44-66

Augspurger CK (1981) Reproductive synchrony of a tropical shrub: experimental studies on effects of pollinator and seed predators on Hybanthus prunifolius (Violaceae). Ecology 62:775-788

Augspurger CK (1983) Seed dispersal of tropical tree, Platypodium elegans and the escape of its seedlings from fungal pathogens. J Ecol 71:759-771

Bach CS (2002) Phenological patterns in monsoon rainforests in the northern territory, Australia. Austral Ecol 27:477-489

Bhat DM (1992) Phenology of tree species of tropical moist forest of Uttara Kannada district, Karnataka, India. J Biosci 17:325-352

Boojh R, Ramakrishnan PS (1981) Phenology of tree in sub-tropical evergreen montane forest in north - eastern India. Geo Eco Trop 5:189-209

Borchert R (1983) Phenology and control of flowering in tropical trees. Biotropica 15:81-89

Borchert R (1994a) Induction of rehydration and bud break by irrigation or rain in deciduous trees of a tropical dry forest in Costa Rica. Trees 8:198-204

Borchert R (1994b) Soil and stem water storage determine phenology and distribution of tropical dry forest trees. Ecology 75:1437-1449

Borchert R (1998) Response of tropical trees to rainfall seasonality and its long-term changes. Climate Change 39:381-393

Borchert R, Meyer SA, Felger RS, Porter-Bolland L (2004) Environmental control of flowering periodicity in Costa Rican and Mexican tropical dry forests. Glob EcolBiogeogr 13:409-425

Bullock SH, Solis-Magallanes JA (1990) Phenology of canopy trees of a tropical deciduous forest in México. Biotropica 22:22-35

Burger WC (1974) Flowering periodicity at four altitudinal levels in eastern Ethiopia. Biotropica 6:38-42

Champion HG, Seth SK (1968) A Revised Survey of the Forest Types of India. Govternment of India Press, Howrah, p 404

Chapman CA, Wrangham RW, Chapman JJ, Kennard DK, Zanne AE (1999) Fruit and flower phenology at two sites in Kibale National Park, Uganda. J Trop Ecol 15:189-211

Chapman CA, Chapman LJ, Struhsaker TT, Zanne AE, Clark CJ, Poulsen JR (2005) A long-term evaluation of fruiting phenology: importance of climate change. J Trop Ecol 21:31-45

Coley PD, Barone JA (1996) Herbivory and plant defenses in tropical forests. Annu Rev EcolSyst 27:305-335

Daubenmire R (1972) Phenology and other characteristics of tropical semideciduous forest in north - western Costa Rica. J Ecol 60:147-170

Eamus D, Prior L (2001) Ecophysiology of trees of seasonally dry tropics: Comparisons among phenologies. Adv Ecol Res 32:113-197

Elliot S, Baker PJ, Borchert R (2006) Leaf-flushing during the dry season: the paradox of Asian monsoon forests. Glob EcolBiogeogr 15:248-257

Estrella N, Menzel A (2006) Responses of leaf colouring in four deciduous tree species to climate and weather in Germany. Climate Res 32:253-267 
Foster RB (1982) The seasonal rhythms of fruit fall on Barro Colorado Island. In: Leigh EG, Rand AS, Windsor DM (eds) The Ecology of a Tropical forest: Seasonal Rhythms and Long-Term Changes. Smithsonian Institution Press, Washington, DC

Frankie GW, Baker HG, Opler PA (1974) Comparative phenological studies of trees in tropical wet and dry forests in the lowlands of Costa Rica. J Ecol 62:881-919

Gamble JS, Fischer CEC (1998) Flora of the Presidency of Madras. Volumes 1, 2 and3. Adlard and Son Ltd, London, p 2017

Gentry AH (1974) Flowering phenology and diversity in tropical Bignoniaceae. Biotropica 6:64-68

Heideman PD (1989) Temporal and spatial variation in the phenology of flowering and fruiting in a tropical rainforest. J Ecol 77:1059-1079

Janzen DH (1967) Synchronization of sexual reproduction of trees within the dry season in Central America. Evolution 21:620-637

Justiniano MJ, Fredericksen TS (2000) Phenology of tree species in Bolivian dry forests. Biotropica 32:276-281

Keskitalo J, Bergquist G, Gardestrom P, Jansson S (2005) A cellular timetable of autumn senescence. Plant Physiol 139:1635-1648

Koike T (1990) Autumn coloring, photosynthetic performance and leaf development of deciduous broad-leaved trees in relation to forest succession. Tree Physiol 7:21-32

Krishnamurthy YL, Prakasha HM, Nanda A (2009) Floristic diversity of Bhadra Wildlife Sanctuary in the Central Western Ghats region, Karnataka. The Indian Forester 135:1397-1406

Krishnamurthy YL, Prakasha HM, Nanda A, Krishnappa M, Dattaraja HS, Suresh HS (2010) Vegetation structure and floristic composition of a tropical dry deciduous forest in Bhadra Wildlife Sanctuary, Karnataka, India. Trop Ecol 51:235-246

Krishnan RM (2002) Reproductive phenology of a wet forest understorey in the Western Ghats, south India. Glob EcolBiogeogr 11:179-182

Lieberman D (1982) Seasonality and phenology in a dry tropical forest in Ghana. J Ecol 70:791-806

Marquis RJ (1988) Phenological variation in the neotropical understorey shrub Piper arieianum: causes and consequences. Ecology 69:1552-1565

Mooney HA, Medina E, Bullock SH (1995) Neotropical Deciduous Forests. Academic Press, New York

Morellato LPC, Talora DC, Takahasi A, Becke CC, Romera EC, Zipparro VB (2000) Phenology of Atlantic rain forest trees: a comparative study. Biotropica 32:811-823

Murali KS, Sukumar R (1993) Leaf flushing phenology and herbivory in a tropical dry deciduous forest, southern India. Oecologia 94:114-119

Murali KS, Sukumar R (1994) Reproductive phenology of a tropical dry forest in Mudumalai, Southern India. J Ecol 82:759-767

Murray KG, Feinsinger P, Busby WH, Linhart YB, Beach JH, Kinsman S (1987) Evaluation of character displacement among plants in two tropical pollination guilds. Ecology 68:1283-1293

Nanda A (2009) Vegetative and Reproductive Phenology of Tropical Forest Communities in the Areas of Bhadra Wildlife Sanctuary, Karnataka, PhD Thesis. Kuvempu University, Karnataka, p 183

Nanda A, Prakasha HM, Krishnamurthy YL, Suresh HS (2010) Phenology of a tropical dry forest: study from Bhadra Wildlife Sanctuary, Karnataka, peninsular India. Indian J For 33:167-172

Nanda A, Prakasha HM, Krishnamurthy YL, Suresh HS (2011) Phenology of leaf flushing, flower initiation and fruit maturation in dry deciduous and evergreen forests of Bhadra Wildlife Sanctuary, Karnataka, southern India. Our Nature 9:89-99

Nanda A, Krishnamurthy YL, Suresh HS (2013a) Canopy trees leaf phenology in tropical dry deciduous and evergreen forests of Bhadra Wildlife Sanctuary, Karnataka, India. African J Plant Sci 7(5):170-175

Nanda A, Prakasha HM, Krishnamurthy YL, Suresh HS (2013b) Leaf, flower and fruit bud phenology in canopy and understorey trees of a tropical dry deciduous forest, Bhadra Wildlife Sanctuary, Karnataka, southern India. Ann For 21(1):48-58

Neginhal SG (2004) Forest Trees of South India. Navbharath Press, Bangalore, p 447

Newstrom LE, Frankie GW, Baker HG (1994) A new classification for plant phenology based on flowering patterns in lowland tropical rainforest trees at La Selva, Costa Rica. Biotropica 2:141-159

Parameshwar G (1996) Draft Management Plan for Bhadra Wildlife Sanctuary (1996-2001) Publication: Wildlife Division. Chikmagalur, Karnataka Forest Department, Chikmagalur, Karnataka, India

Prakasha HM, Nanda A, Krishnamurthy YL (2008) Stand structure of a tropical dry deciduous forest in Bhadra wildlife sanctuary, Karnataka, Southern India. Bulletin of the National Institute of Ecology. Int J Ecol Environ Sci 19:1-7
Prasad SN, Hegde M (1986) Phenology and seasonality in the tropical deciduous forest of Bandipur, South India. Proceedings Indian Academy Sciences (Plant Science) 96:121-133

Prasad SN, Sharatchandra HC (1984) Primary production and consumption in the deciduous forest ecosystems of Bandipur in South India. Proceedings Indian Academy Science (Plant Science) 93:83-97

Ramaswamy SN, Rao MR, Govindappa DA (2001) Flora of Shimoga District Karnataka. Prasaranga University of Mysore, Manasagangotri, Mysore, p753

Rathcke B, Lacey EP (1985) Phenological patterns of terresterial plants. Ann Rev Ecolsyt 16:179-214

Rivera GE, Caldas LS, Nicolossig Coradin VTR, Orchertr B (2002) Increasing day-length induces spring flushing of tropical dry forest trees in the absence of rain. Trees 16:445-456

Robertson C (1895) The philosophy of flower seasons, and the phenological relations of the entomophilous flora and the anthophilous insect fauna. Am Nat 29:97-117

Sakai S (2001) Phenological diversity in tropical forests. Popul Ecol 43:77-86

Sakai S, Momose K, Yumoto T, Nagamitsu T, Nagamasu H, Hamid AA, Nakashizuka T (1999) Plant reproductive phenology over four years including an episode of general flowering in a lowland Dipterocarp forest, Sarawak, Malaysia. Am J Bot 86:1414-1436

Saldanha CJ (1996) Flora of Karnataka. Volumes I to IV. Oxford and IBH Publishing Co. Pvt. Ltd, New Delhi, p839

Schongart J, Piedade MTF, Ludwigshausen S, Horna V, Worbes M (2002) Phenology and stem growth periodicity of tree species in Amazonian flood plain forests. J Trop Ecol 18:581-597

Singh KP, Kushwaha CP (2005) Emerging paradigms of tree phenology in dry tropics. Curr Sci 89:964-975

Singh JS, Singh VK (1992) Phenology of seasonally dry tropical forest. Curr Sci 63:684-688

Singh KP, Singh JS (1998) Certain structural and functional aspects of dry tropical forest and savanna. Int J Ecol Environ Sci 14:31-45

Snow DW (1965) A possible selective factor in the evolution of fruiting seasons in tropical forest. Oikos 15:274-281

Stiles FG (1975) Ecology, flowering phenology, and hummingbird pollination of some Costa Rican Heliconia species. Ecology 56:285-301

Sun C, Kaplin BA, Kristensen KA, Munyaligoga V, Mvukiyumwami J, Kajondo KK, Moermond TC (1996) Tree phenology in a tropical montane forest in Rwanda. Biotropica 28:668-681

Sundrapandian SM, Chandrasekaran S, Swamy PS (2005) Phenological behaviour of selected tree species in tropical forests at Kodayar in the Western Ghats, Tamil Nadu, India. Curr Sci 88:805-810

Suresh HS, Sukumar R (2011) Vegetative phenology of tropical montane forests in the Nilgiris, South India. J NatnSci Foundation Sri Lanka 39(4):337-347

Van Schaik CP (1986) Phenological changes in a Sumatran rain forest. J Trop Ecol 2:327-347

Van Schaik CP, Terborgh JW, Wright SJ (1993) The phenology of tropical forests: Adaptive significance and consequences for primary consumers. Annu Rev Ecolsyst 24:353-377

Whitmore TC (1996) An Introduction to Tropical Rain Forests. Clarendon Press, Oxford

Williams-Linera G (1997) Phenology of deciduous and broad leaf evergreen tree species in a Mexican tropical lower montane forest. Glob EcolBiogeogrLett 6:115-127

Wright SJ (1996) Phenological responses to seasonality in tropical forest plants. In: Mulkey SD, Chazdon RL, Smith AP (eds) Tropical Forest plant Ecophysiology. Chapman and Hall, New York, pp 440-460

Wright SJ, Van Schaik CP (1994) Light and the phenology of tropical trees. Am Nat 143:192-199

Yasuda M, Matsumoto J, Osada N, Ichikawa S, Kachi N, Tani M, Okuda T, Furukawa A, Nik AR, Manocaran N (1999) The Mechanism of general flowering in Diptercarpaceae in the Malay Peninsula. J Trop Ecol 15:437-449

Yoganarasimhan SN, Subramanyam K, Razi BA (1982) Flora of Chikkamagalur District. Karnataka. India International Book Distributors, Deharadun, p 407 Zar J (2007) Biostatistical Analysis. Pearson Education, London, 663

\section{doi:10.1186/2192-1709-3-1}

Cite this article as: Nanda et al:: Phenology of a tropical dry deciduous forest of Bhadra wildlife sanctuary, southern India. Ecological Processes 2014 3:1. 